International Journal of Engineering \& Technology, $7(4)(2018) 2800-2805$
International Journal of Engineering \& Technology
SPC
Website: www.sciencepubco.com/index.php/IJET
doi: $10.14419 /$ ijet.v7i4.18089
Research paper

\title{
Adopting pade approximation for first order plus dead time models for blending process
}

\author{
Avani Kirit Mehta ${ }^{1}$, R. Swarnalatha ${ }^{2} *$ \\ ${ }^{I}$ Dept of Electronics \& Instrumentation Engineering, Birla Institute of Technology \& Science, Pilani, Dubai Campus, Dubai. U.A.E \\ ${ }^{2}$ Assistant Professor, Dept of EEE, Birla Institute of Technology \& Science, Pilani, Dubai Campus, Dubai. U.A.E \\ *Corresponding author E-mail: swarnalatha@dubai.bits-pilani.ac.in
}

\begin{abstract}
Dead-time is common to real time processes and occurs when the process variable doesn't acknowledge to any changes in the set point. Existence of dead time in the systems poses a challenge to control and stabilize, especially in a control feedback loop. Padé approximation provides a determinate approximation of the dead time in the continuous process systems, which can be utilized in the further simulations of equivalent First Order plus Dead Time Models. However, the standard Padé approximation with the same numerator- denominator derivative power, exhibits a jolt at time $\mathrm{t}=0$. This gives an inaccurate approximation of the dead time. To avoid this phenomenon, increasing orders of Padé approximation is applied. In the following manuscript, equivalent First Order plus Dead-Time models of two blending systems of orders four and seven are analysed for the same. As the orders of the Padé approximation increases, the accuracy of the response also increases. The oscillations are increased on a much smaller scale rather than having one big dip in the negative region (as observed in the first few orders of Padé approximation), and the approximation tries to synchronize with the desired response curve in the positive region. All the simulations are done in MATLAB.
\end{abstract}

Keywords: First Order Pus Dead Time; Process Dead Time; Padé Approximation; Process Gain Constant; Two Point Method of Approximation

\section{Introduction}

Blending systems are very common in process industries such as Oil \& Gas, Waste water treatment, Paper, Food, Pharmaceutical, Chemical and many more (Saravanakumar and Wahidabanu, 2009). The real time industrial problems are non-linear in nature and exhibit dead time / time delay (Smith et al, 2006). This dead time occurs majorly due to the following factors (Bequette, 2002):

1) External factors such as transportation lag due to long pipelines or large travel distances.

2) Internal factors such as non linearities of the Final Control Element, i.e., blunt use of conventional actuator sizing for valves, and excessive tuning of the controller.

3) Uncertainties like noisy data, erroneous assumptions of important parameters, and incorrect modelling of the systems.

Uncertainties occur in the following forms (Rhinehart, 2016):

1) Parametric uncertainty where the incorrect parameters are communicated

2) Model uncertainty where wrong modelling of the process/system under consideration is done

3) Stochastic uncertainty where the modelled outcome deviates a great degree from the expected outcome, given there is no Parametric or Model Uncertainties.

Presence of dead time element complicates the analysis and design of control systems and makes satisfactory control more difficult as the performance might endure instability, high sensitivity to parametric uncertainties and poor disturbance rejection (Rhinehart, 2016). One of the focal consequences of dead time include the effect of disturbances not seen by the controller for a while, thus making the effect of control action non-existent at the output caus- ing the controller to take additional compensation unnecessarily, thus resulting in a loop with limitations to control (Bequette, 2002).

Any industrial process is mathematically represented in the form of nonlinear differential equations (continuous domain) or difference equations (discrete domain). Using analytical methods such as State Space Analysis, Initial-Final Value theorems etc. to solve these equations become a challenge with the increasing nonlinearities, orders of the transfer functions and dead time (Nidhi Yadav and Chandra Shekhar, 2017). The FOPDT model is often an equitable approximation to such process behaviours, as it has the efficacy for controller tuning rules and can be used as a computationally surrogate model in simulations for training and optimization (Korsane et al, 2014). Higher order industrial processes can be modelled as FOPDT, as the simulations become much easier.

The FOPDT model has the continuous transfer function (Eq. 1) (Bequette, 2002):

$$
\frac{K p}{\tau s+1} \cdot e^{-\theta s}
$$

Where: Kp: Process Gain

$\tau$ : Process Time constant

$\Theta$ : Process Dead Time

(Eq. 2) (Bequette, 2002) gives the transfer function for a pure time delay:

$T(s)=e^{-\theta s}$ 
Some control system designs require a rational transfer function and the Padé approximation provides a determinate approximation of the dead-time in the continuous process systems, which can be utilized in the further simulations of equivalent First Order Plus Dead Time Models (Pradeep et al., 2010).However, the standard Padé approximation with the same numerator- denominator derivative power, exhibits a jolt at time $\mathrm{t}=0$. This gives an inaccurate approximation of the dead time (Palmor and Z. J, 1996). To avoid this phenomenon, increasing orders of Padé approximation is applied (Kano and Ogawa, 2010).

(Eq. 3) gives a first-order Padé approximation:

$e^{-\theta s}=\frac{-\theta / 2^{s+1}}{\theta / 2 s+1}$

(Eq. 4) gives a second-order Padé approximation:

$$
e^{-\theta s}=\frac{-\theta^{2} / 12^{s^{2}-\theta / 2} s+1}{\theta^{2} / 12^{2}+\theta / 2 s+1}
$$

\section{Materials and methods}

Two transfer functions, fourth order (Eq. 5) and seventh order (Eq. 6) mimicking Blending processes have been used for experimentation (Shahian and Hassul, 1993).

$$
G(s)=\frac{1}{(10 s+1)(s+1)(0.1 s+1)(0.05 s+1)}
$$

$$
T(s)=\frac{1}{(s+1)^{7}}
$$

The two point method of approximation uses the formulations given below. The controller gain is calculated using (Eq. 7).

$\mathrm{Kc}=$ change in output/ change in input

The process time constant is given by (Eq. 6).

The process time constant, which is the time required to obtain $63.2 \%$ of the final steady state value is given by (Eq. 8 )

$\tau_{p}=1.5\left(t_{0.632}-t_{0.283}\right)$

The process dead time is calculated using (Eq. 9).

$$
\theta=t_{0.632}-\tau_{p}
$$

Finally the FOPDT model is obtained using the (Eq. 1).

Using the above formulations, the FOPDT models for 4th (Eq. 10) and 7th (Eq. 11) orders are given as follows (Z. Kalateh et al., 2013):

$$
\begin{aligned}
& P(s)=\frac{e^{-1.15 s}}{10 s+1} \\
& Q(s)=\frac{e^{-4.16 s}}{3.417 s+1}
\end{aligned}
$$

The following block diagram is (Fig. 1) is used up for simulations related to the FOPDT modelling of the 4th order blending process (Eq. 5) \& (Eq. 10) to obtain the responses for a unit step input.

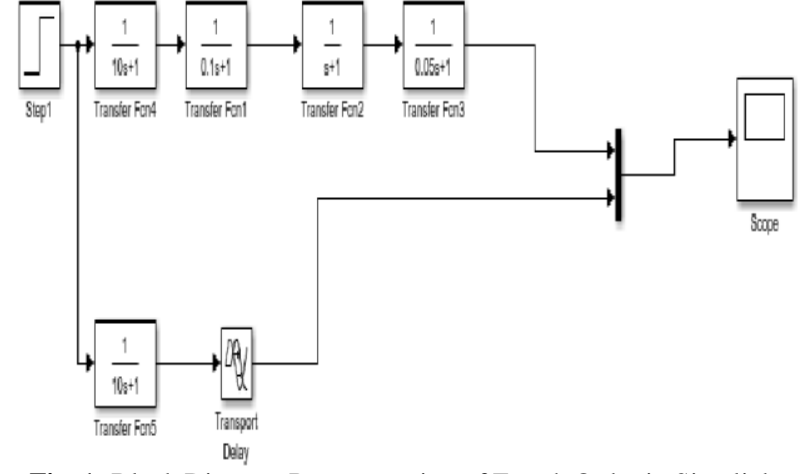

Fig. 1: Block Diagram Representation of Fourth Order in Simulink.

(Fig. 2) shows the response of the fourth order transfer function model and FOPDT model for a unit step input. Both the graphs are approximately the same; therefore the approximation done using two-point method is correct.

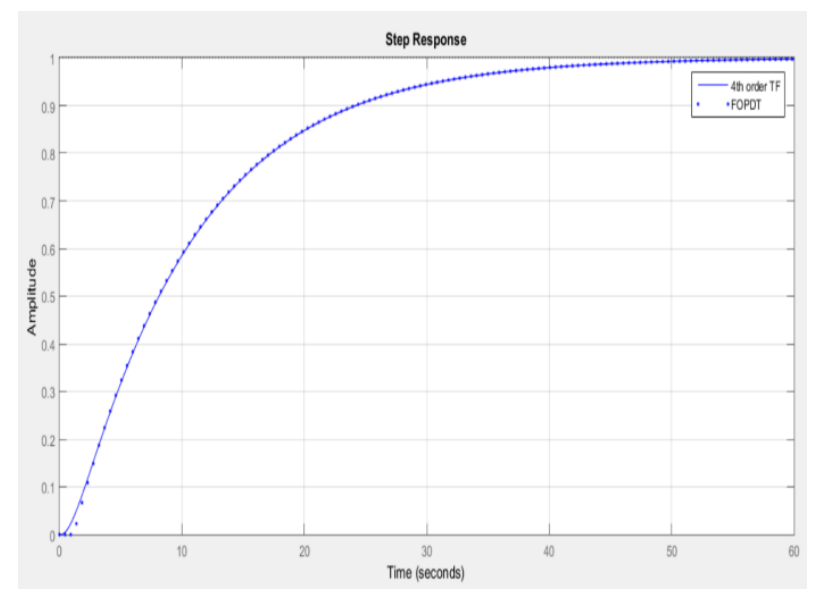

Fig. 2: Response of fourth Order Transfer Function and Dead Time Approximation.

(Fig. 3) is used up for simulations for $7^{\text {th }}$ order blending process represented by (Eq. 6) \& (Eq. 11) and their responses have been obtained.

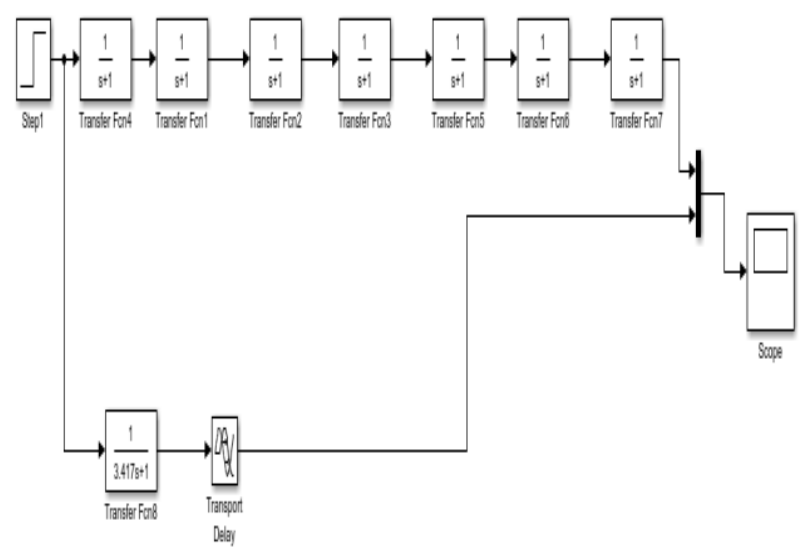

Fig. 3: Block Diagram Representation of Seventh Order in Simulink.

(Fig. 4) shows the response of the seventh order transfer function model and FOPDT model obtained using the two-point method of approximation. 


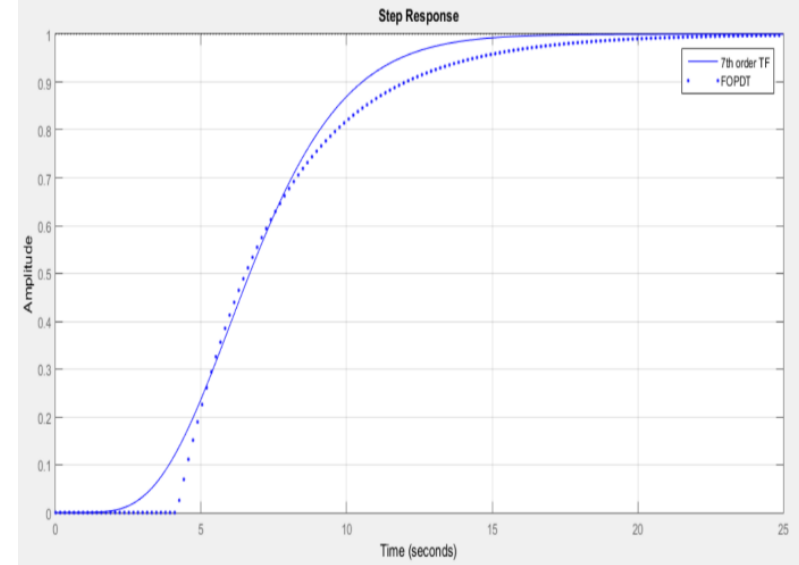

Fig. 4: Response of seventh Order Transfer Function and Dead Time Approximation.

\section{Results \& discussions}

The following graphs for $4^{\text {th }}$ and $7^{\text {th }}$ order systems modelled as FOPDT have been obtained for a unit step as the input.

\subsection{Fourth order}

i) First order Padé Approximation:

The Padé approximation for first order is (Eq. 12):

$$
\frac{-s+1.739}{10 s^{2}+18.39 s+1.739}
$$

From Fig. 5, a jolt at $\mathrm{t}=0 \mathrm{~s}$ in the negative region is observed. This happens due to the zero in the right-half plane and this observation is highly undesired as it gives an inaccurate approximation. In addition, this response is inaccurate in the high frequency region and accurate in the low frequency region (Juneja et al, 2010).

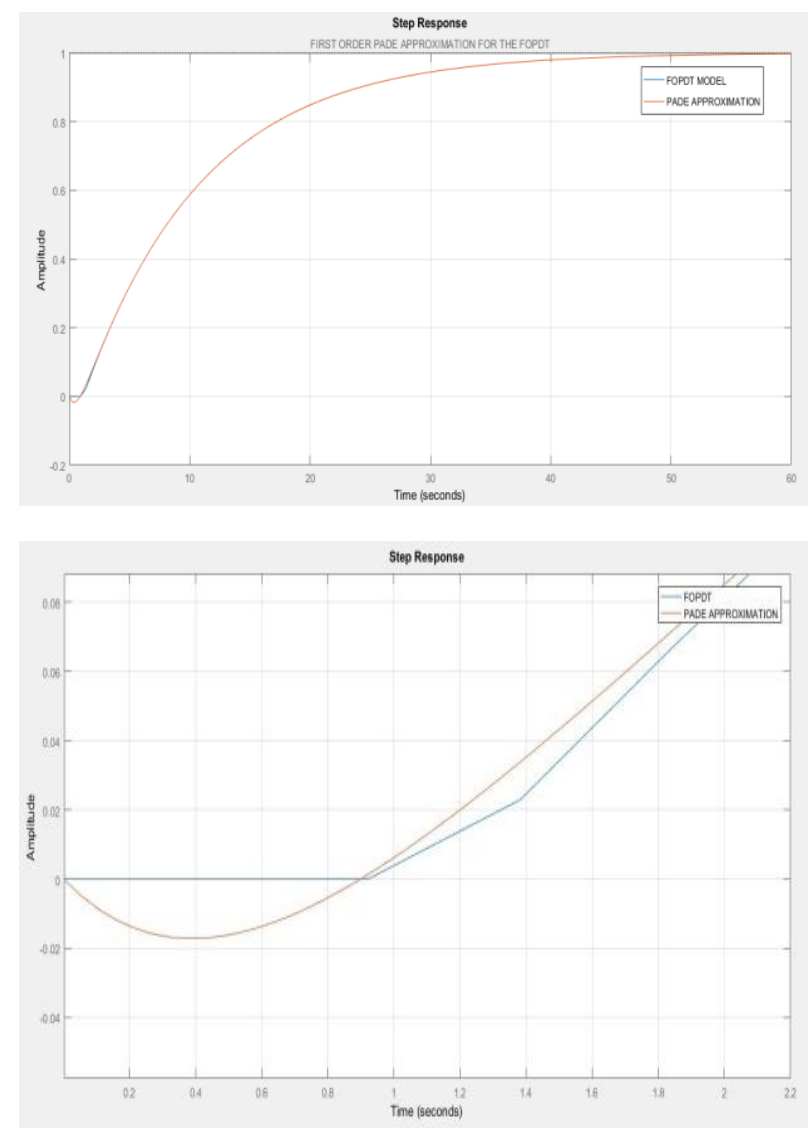

Fig. 5: Response with First Order Padé Approximation
The Padé approximation for second order is (Eq. 13):

$\frac{s^{2}-5.217 s+9.074}{10 s^{3}+53.17 s^{2}+95.95 s+9.074}$

The Padé approximation for fifth order is (Eq. 14):

$\frac{-\mathrm{s}^{5}+26.09 \mathrm{~s}^{4}-317.6 \mathrm{~s}^{3}+2209 \mathrm{~s}^{2}-8645 \mathrm{~s}+1.503 \mathrm{e}^{4}}{10 \mathrm{~s}^{6}+261.9 \mathrm{~s}^{5}+3202 \mathrm{~s}^{4}+2.24 \mathrm{e}^{4} \mathrm{~s}^{3}+8.8663 \mathrm{e}^{4} \mathrm{~s}^{2}+1.59 \mathrm{e}^{4} \mathrm{~s}+1.503 \mathrm{e}^{4}}$

As very evident from the Eq. 13 \& Eq. 14, as the order of pade approximation increases, the number of terms also increases. Thus we can assume that the complexity in the analytical aspect of the system also increases. The response graphs for the increasing orders of Pade's approximation for a unit step have been obtained in the following figures.

ii) Twenty-seventh order Padé Approximation

(Eq. 15) gives the Padé approximation for 27th order:

$\frac{-s^{27}+657.4 s^{26}-2.155 e^{5} s^{25}+\ldots \ldots . .2 .8 e^{41} s+4.87 e^{41}}{10 s^{28}+6575 s^{27}+\ldots \ldots . .+2.87 e^{42} s^{2}+5.15 e^{42} s+4.87 e^{41}}$

The following figure gives the response for the $27^{\text {th }}$ order of Padé approximation.

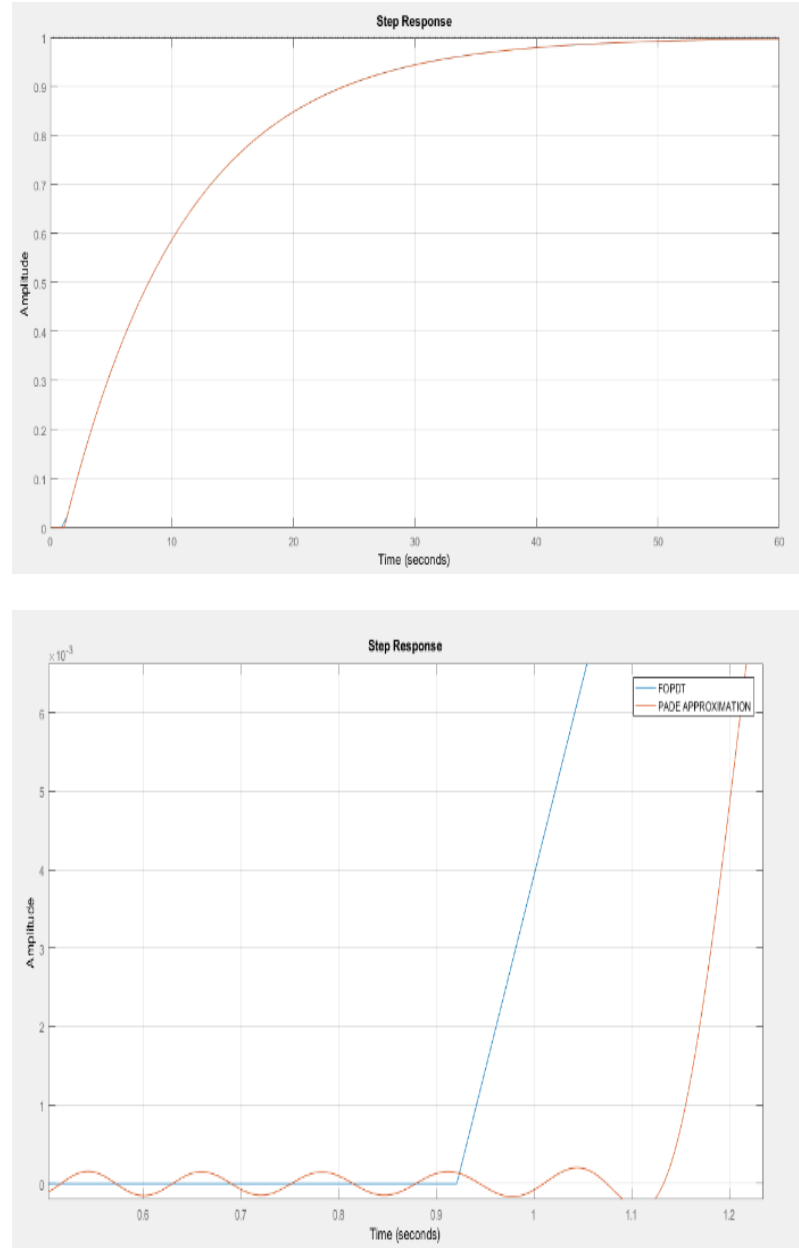

Fig. 6: Response with Twenty-Seventh Order Padé Approximation

iii) Fiftieth order Padé Approximation: 

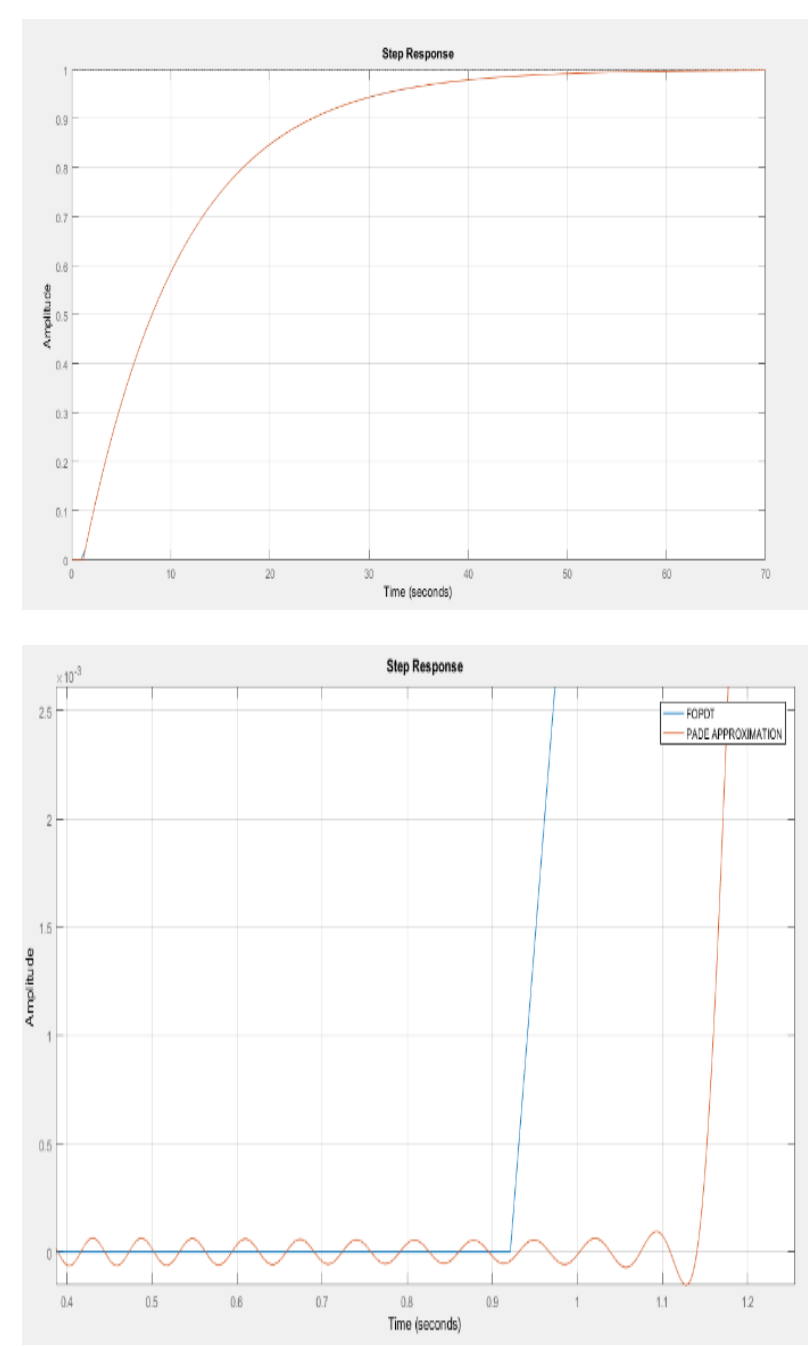

Fig. 7: Response with Fiftieth Order Padé Approximation.

(Eq. 16) gives the Padé approximation for 50th order and the response is obtained in Fig. 7.

$\frac{s^{50}-2217 s^{49}+2.456 * 10^{6} s^{48}+\ldots .+4.634 * 10^{89} s^{2}-1.628 * 10^{90} s+2.832 * 10^{90}}{10 s^{51}+2.217 * 104 s^{50}+\ldots .+1.675 * 10^{91} s^{2}+2.994 * 10^{91} s+2.832 * 10^{90}}$

iv) Seventy-seventh order Padé Approximation

The Padé approximation for $77^{\text {th }}$ order is given by (Eq. 17) and the response is obtained in Fig. 8.

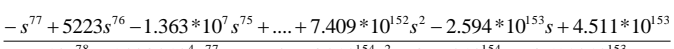
$10 s^{78}+5.223 * 10^{4} s^{77}+\ldots .+2.668 * 10^{154} s^{2}+4.77 * 10^{154} s+4.511 * 10^{153}$

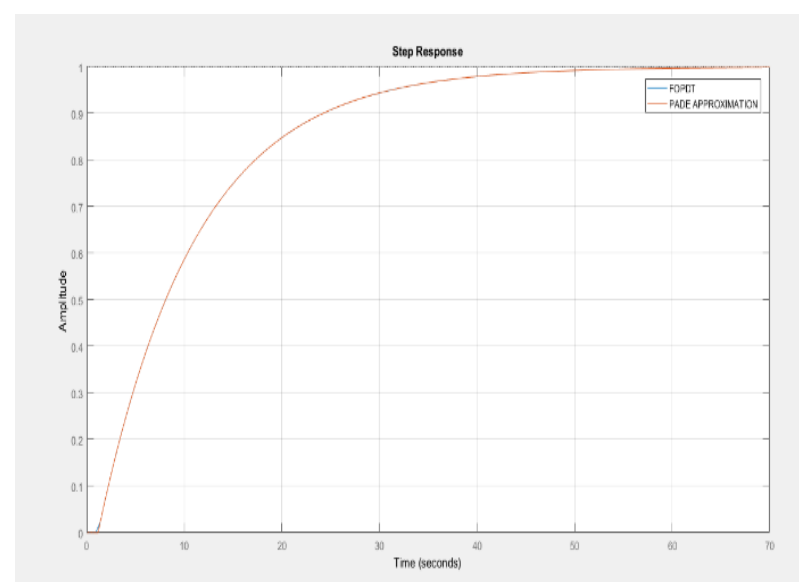

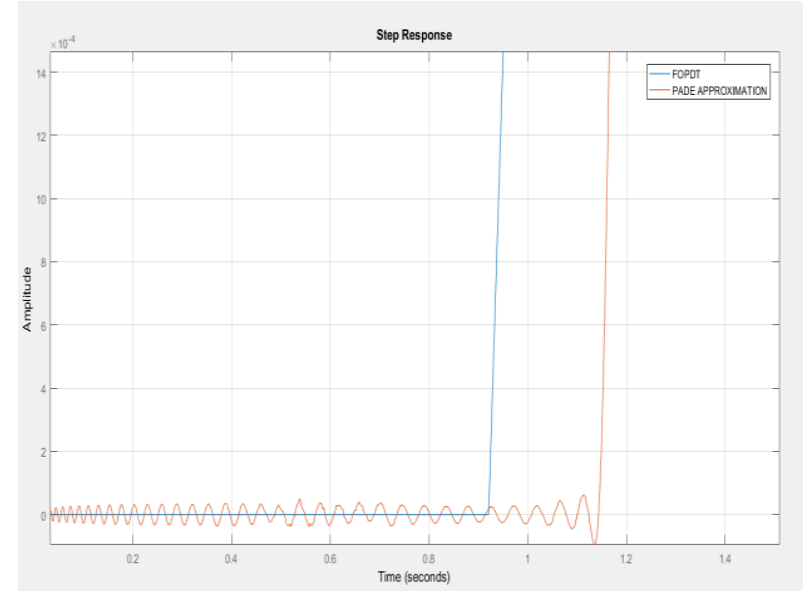

Fig. 8: Response with Seventy-Seventh Order Padé Approximation.

As the orders of the Padé approximation increases, the accuracy of the response also increases. The oscillations are increased on a much smaller scale rather than having one big dip in the negative region (as observed in the first few orders of Padé approximation), and the approximation tries to sync with the desired FOPDT response curve in the positive region. Clearly, these are more accurate approximations when compared to the first few orders of Padé approximation. The response oscillates several times during the dead time period by small amplitude, thus giving a good approximation to dead time. On the other hand, the expression for the transfer function is quite complex and is not as useful for algebraic manipulation as is the first order approximation.

v) Seventy-eighth order Padé Approximation:

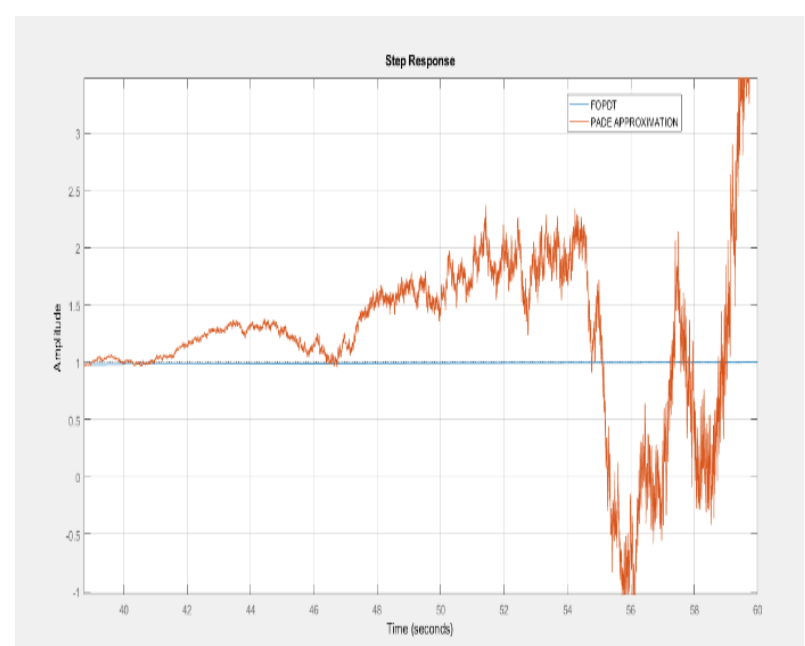

Fig. 9: Response with Seventy-Eighth Order Padé Approximation.

Note that the instability in the response arises immediately at the $78^{\text {th }}$ order of the Padé approximation, and continue from herewith, for the $4^{\text {th }}$ order equivalent FOPDT model. This helps us conclude that Padé approximation beyond $77^{\text {th }}$ order does not yield a stable accurate result.

\subsection{Seventh order}

i) First order Padé Approximation:

(Eq. 17) gives the Padé approximation for first order:

$\frac{-\mathrm{s}+0.4808}{3.417 \mathrm{~s}^{2}+2.643 \mathrm{~s}+0.4808}$

A similar large dip (as also observed in the case of fourth order system) deviating from the FOPDT response curve is observed in the Padé approximation response curve towards the negative region for the seventh order system modelled as FOPDT. 


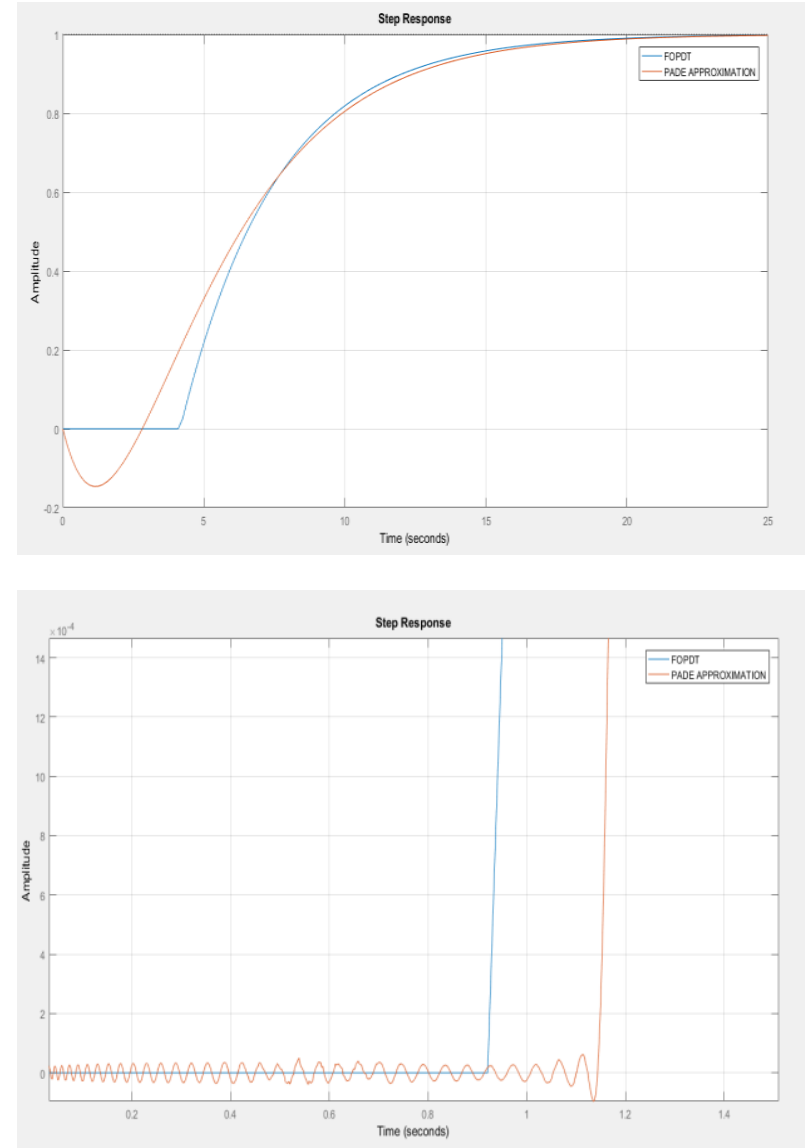

Fig. 10: Response with First Order Padé Approximation.

(Eq. 18) gives the Padé approximation for second order:

$\mathrm{s}^{2}-1.442 \mathrm{~s}+0.6934$

$3.417 \mathrm{~s}^{3}+5.928 \mathrm{~s}^{2}+3.812 \mathrm{~s}+0.6934$

The Padé approximation for sixth order is (Eq. 19):

$s^{6}-10.1 s^{5}+48.54 s^{4}-140 s^{3}+252.4 s^{2}-267 s+128.4$

$3.417 \mathrm{~s}^{7}+35.5 \mathrm{~s}^{6}+176 \mathrm{~s}^{5}+527 \mathrm{~s}^{4}+1003 \mathrm{~s}^{3}+1165 \mathrm{~s}^{2}+705.6 \mathrm{~s}+128.4$

ii) Fortieth order Padé Approximation:

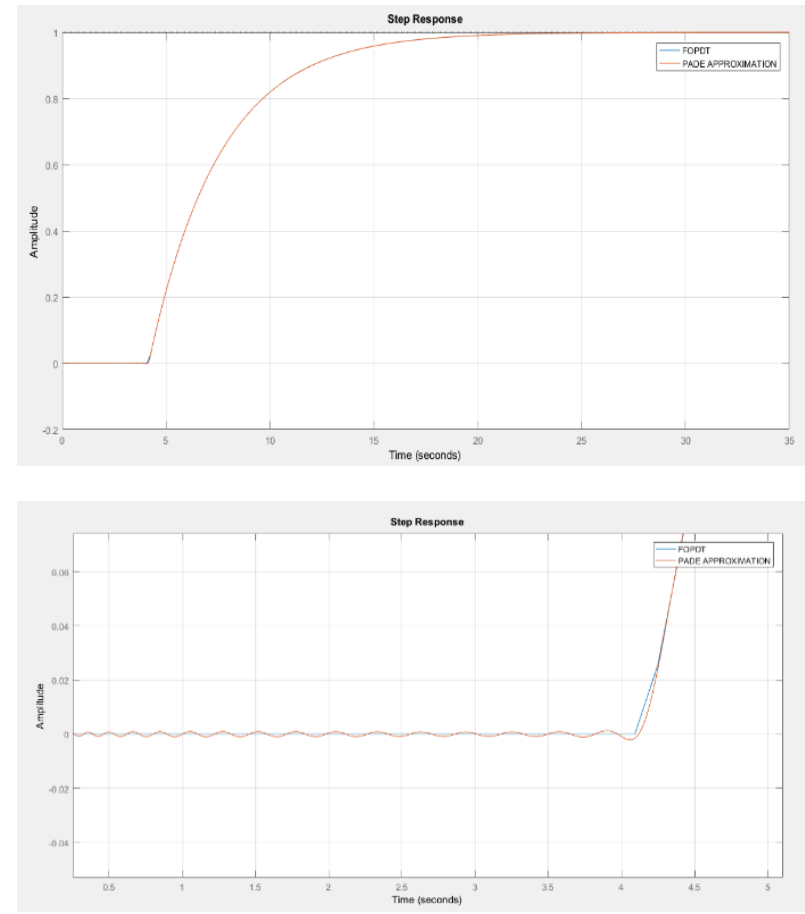

Fig. 11: Response with Fortieth Order Padé Approximation. iii) Seventy-eighth order Padé Approximation:

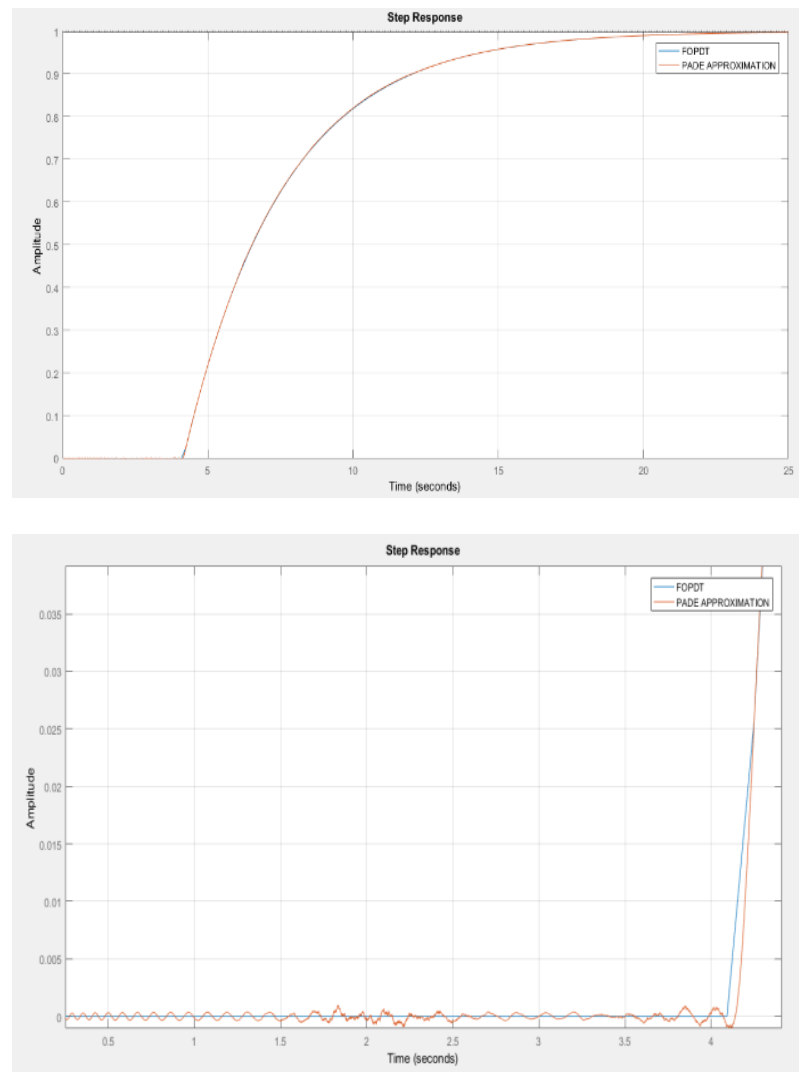

Fig. 12: Response with Seventy-Eighth Order Padé Approximation.

As also observed in the case of fourth order system, on increasing the order of Padé approximation, more accurate responses have been obtained in the case of seventh order system as well. The oscillations are increased on a much smaller scale rather than having one big dip in the negative region (as observed in the first few orders of Padé approximation), and the approximation tries to synchronize with the desired FOPDT response curve in the positive region.

iv) Seventy-nineth order Padé Approximation:

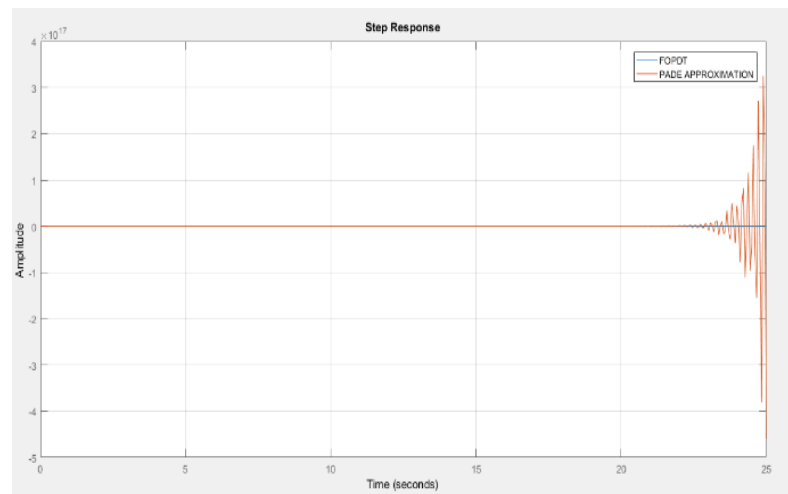

Fig. 13: Response with Seventy-Nineth Order Padé Approximation.

Note that the instability for the seventh order blending process modelled as FOPDT, in the response arises immediately at the $79^{\text {th }}$ order of the Padé approximation, and continue from herewith, for the $7^{\text {th }}$ order equivalent FOPDT model. This helps us conclude that Padé approximation beyond $78^{\text {th }}$ order does not yield a stable accurate result.

\section{Conclusions}

For both the order of the blending system- $4^{\text {th }}$ and $7^{\text {th }}$ order, similar responses have been obtained, i.e., as the order of the Padé approximation was increased, more accuracy in the FOPDT re- 
sponse was obtained with minimum deviation from the transfer function response. The oscillations are increased on a much smaller scale rather than having one big dip in the negative region, as was observed in the first few orders of Padé approximation, and the approximation eventually tries to sync with the desired FOPDT response curve in the positive region.

The Pade's approximation of first order, second order or higher can thus provide a rational transfer function for various situations and is very useful in control analysis. Its accuracy is more evident at higher frequencies with larger orders of the approximation. The trade-off, however, is that as the mathematical expression becomes more complicated, it is more difficult to gain insight from the expression analytically, even though the numerical results are more accurate.

\section{Acknowledgement}

The authors would sincerely like to thank Prof. R. N. Saha, Director, BITS Pilani, Dubai Campus for his constant encouragement and support.

\section{References}

[1] Coughanowr D. R., Process Systems Analysis and Control, McGraw-Hill International Edition, Chemical Engineering Series (1991)

[2] Kano, M.; Ogawa, M., The state of art in chemical process control in Japan: Good practice and questionnaire survey. J. Process Control 2010, 20, 969-982. https://doi.org/10.1016/j.jprocont.2010.06.013.

[3] Korsane, D.T., V. Yadav and K.H. Raut (2014) 'PID tuning rules for first order plus time delay system', Int. J. Innov. Res. Electr. Electron. Instrumentation Control Eng., vol. 2: 582-586.

[4] Morari, M. and Zafiriou, E., Robust Process Control, Prentice-Hall, Englewood Cliffs, NJ, 1989

[5] Nidhi Yadav and Chandra Shekhar, Comparison of PID Controller Tuning Methods for FOPDT and SOPDT of Unstable System, Intnl. Journal of Innovative Research in Science and Technology, Vol.No.3, Issue 02, February 2017, ISSN (0) 2454-9665.

[6] Palmor, Z. J.: Time-delay compensation Smith predictor and its modifications. In W. Levine, editor, The Control Handbook. CRC Press, Boca Raton, FL, (1996).

[7] Pradeep Kumar Juneja, A. K. Ray \& R. Mitra, Dead time Modeling for First Order Plus Dead Time Process in a Process Industry, International Journal of Computer Science \& CommunicationVol. One, No. 2, July-December 2010, pp. 167-169.

[8] Qing-Chang Zhong and Leonid Mirkin, Control of Integral Processes with Dead Time Part II: Quantitative Analysis, IEE Proc.Control Theory Appl. Vol 149, No. 4, July 2002, pp.291-296.

[9] Reshma Babu and R. Swarnalatha, 2017. Comparison of Different Tuning Methods for $\mathrm{pH}$ Neutralization in Textile Industry. Journal of Applied $\quad$ Sciences, 17: 142-147. https://doi.org/10.3923/jas.2017.142.147.

[10] Rhinehart, R. (2016, December 8). Testing a First-Order plus Dead time model. Retrieved from https://www.controlglobal.com/articles/2016/testing-a-first-orderplus-deadtime-model/.

[11] Saravanakumar, G. and Wahidabanu, R. S. D. Control of integrating process with dead time using auto-tuning approach. Braz. J. Chem. Eng., Mar 2009, vol.26, no.1, p.89-98. ISSN 0104-6632

[12] Seborg, Edgar, Mellichap, Doyle, Process Dynamics and control; 2011; Edition 3rd page no. 223-225.

[13] Shahian, B., \& Hassul, M., Control system design using MATLAB Englewood Cliffs, 1993; NJ: Prentice Hall.

[14] Wayne B. Bequette, Process control: Modeling, Design and Simulation, Prentice Hall Professional, First Edn, ISBN-10: 0133536408, 2002.

[15] Z. Kalateh Bojdi, S. Ahmadi-Asl, and A. Aminataei, A New Extended Padé Approximation and Its Application, Hindawi Publishing Corporation Advances in Numerical Analysis Volume 2013 Article ID 263467, 8 pages https://doi.org/10.1155/2013/263467. 